\title{
An Elliptic Current Operator for the Eight Vertex Model
}

\section{Klaus Fabricius*}

Fachbereich C, Bergische Universität Wuppertal

D-42097 Wuppertal

E-mail: Fabriciusetheorie.physik.uni-wuppertal.de

\section{Barry M. McCoy}

Institute for Theoretical Physics, State University of New York,

Stony Brook, NY 11794-3840

E-mail: mccoy@insti.physics.sunysb.edu

Whereas the tools to determine the eigenvalues of the eight-vertex transfer matrix $T$ are well known there has been until recently incomplete knowledge about the eigenvectors of $T$. We describe the construction of eigenvectors of $T$ corresponding to degenerate eigenvalues and discuss the related hidden elliptic symmetry.

BETHE ANSATZ: 75 YEARS LATER

October 19-21, 2006

Brussels, Belgium

${ }^{*}$ Speaker. 


\section{Introduction}

The eight vertex model was introduced into physics by Baxter [1] as the natural generalization of the six vertex model which satisfies the star triangle equations and because the six vertex model is solved by the same ansatz for eigenvectors which was introduced by Bethe [2] in his famous 1931 computation of the eigenvectors and eigenvalues of the isotropic Heisenberg chain it is a common practice of many authors to present the computation of the eigenvalues and eigenvectors of the eight vertex model as a generalization of the methods of Bethe. Indeed it is for this reason that the 8 vertex model is the topic of many talks at this conference honoring the 75 anniversary of Bethe's original paper.

Nevertheless there are several profound distinctions between the 6 and 8 vertex model which make the methods used to study the properties of the 8 vertex model very different from the methods used in the Bethe's ansatz solution of the 6 vertex model as presented, for example, in the classic 1967 papers by Lieb [3] and by Sutherland, Yang and Yang [4] and the corresponding 1966 papers of Yang and Yang [5] for the anisotropic Heisenberg model. Perhaps the most visible of these differences in solution is that while the Bethe ansatz for the eigenvectors of the 6 vertex model is valid for all values of the crossing parameter $\eta$ (and the corresponding anisotropy $\Delta$ of the anisotropic Heisenberg chain) a very important role for the solutions of the 8 vertex model given in [1] is played by what is called the "root of unity" condition

$$
2 L \eta=2 m_{1} K+i m_{2} K^{\prime}
$$

where $2 K$ and $2 K^{\prime}$ are the real and imaginary periods of the quasiperiodic functions which characterize the Boltzmann weights of the 8 vertex model. In particular the condition (1.1) is necessary for the original solution of the eigenvalue problem of the 8 vertex model transfer matrix of [1] to hold and for the solution for eigenvectors given in [6]-[8] to hold it is necessary that the related root of unity condition

$$
L \eta=2 m_{1} K+i m_{2} K^{\prime}
$$

be imposed. A study of the eigenvalue problem for generic values of $\eta$ is given in [6] and [9] however none of these classic studies either for roots of unity or for generic values revealed all the properties of the eigenvalue spectrum. In particular it was found in [10] that the condition (1.1) is not sufficient and for the method of [1] to hold we must require in (1.1) with $m_{2}=0$ that for a system with $N$ lattice sites in a chain that the case with $L$ odd and $m_{1}$ even must be excluded for $N>L-1$ if $N$ is even and for all $N$ if $N$ is odd.

The eigenvectors at generic values of $\eta$ are briefly mentioned in [11],[12], and [13] and in the recent paper of Bazhanov and Mangazaeev [14] presented at this conference.

It is the purpose of this paper to present recent progress in the study of the eigenvalues and eigenvectors of the 8 vertex model at the roots of unity (1.1) with $m_{2}=0$ and $L$ is even or both $L$ and $m_{1}$ are odd for even values of $N$. We found in [10] that one has to distinguish two types of eigenstates of $T$ :

1. singlet states for the nondegenerate eigenvalues.

2. states appearing in multiplets for degenerate eigenvalues.

The singlet states are eigenstates of the spin reflection operator. This should be compared with the 
limiting case of the six vertex model where only a few states with $S^{z}=0$ are singlets. Furthermore in the Bethe ansatz treatment of [2]-[5] the eigenvectors of the Hamiltonian are by definition eigenvectors of the operator $S^{z}$ and thus for $S^{z} \neq 0$ none of the Bethe states can be an eigenstate of the spin reflection operator.

Little attention has usually been paid to the second case despite the fact that depending on the size $N$ and the value of the crossing parameter $\eta$ the overwhelming majority of states is degenerate. As an illustration we mention that for $N=12$ and $\eta=K / 2$ there are 128 non degenerate states in the eigenspace of dimension 4096. This same phenomenon of degeneracies at roots of unity which are larger than the symmetry required under $S^{z} \rightarrow-S^{z}$ occurs in the six-vertex model where it is explained by the existence of an $s l_{2}$ loop algebra [15]. The explanation of the multiplets as highest weight representations of this algebra based on the algebraic Bethe ansatz of [11] was first given in [16] and a rigorous proof in terms of representation theory is given in [17]. A treatment in terms of the coordinate Bethe ansatz is given in [13].

The more complicated problem of obtaining all degenerate eigenstates of $T$ in the algebraic Bethe-Ansatz of the eight-vertex model has been solved recently in [18]. A parallel investigation for the description of the eight-vertex model given in [12] is done in [19, 20].

\section{Degenerate eigenvectors of $T$ in the eight-vertex model.}

Our goal is to find a general procedure allowing the construction of all degenerate eigenstates of the transfer matrix $T$. The powerful and elegant algebraic Bethe-Ansatz [11] is the ideal tool to develop the analytic framework in a transparent manner. Furthermore it will allow the interpretation of the result in the context of symmetry algebras.

We shall first describe the important results obtained in [11]. For the six vertex model the authors find that eigenstates of $T$ are given by a product of $B$-operators acting on a reference state. In the six-vertex model this works for all irrational crossing parameters. For rational multiples of $\pi$ not all eigenstates are obtained but only those which are highest weight states of loop algebra multiplets. To construct the remaining elements of these multiplets in addition to $B$-operators the more complicated creation operators of strings are needed. These creation operators of strings were introduced in [16]. Besides being creation operators of strings they have an important algebraic meaning: They are current operators of the loop algebra symmetry of the six-vertex model. The description of the loop-algebra symmetry of the six-vertex model is given in [15].

In the eight-vertex model the situation is more complicated. First the well developed results $[8,11]$ are valid only at roots of unity. In [11] eigenvectors of $T$ are given by finite sums of products of $B$-operators acting on generalized reference states. Like in the six-vertex model one gets by this method all singlet states. But unlike the case of the six-vertex model the $B$-operators and the reference states of the eight vertex model depend on additional free parameters $s, t$ and by varying these parameters one gets a subspace of dimension larger than one for each degenerate eigenspace. However there still remain a large number of states states in the multiplets which cannot be constructed in this way. For the construction of these missing eigenstates one needs the additional string creation operators which were found in [18]. There are also missing eigenvectors in the solution of the eight-vertex model by Felder and Varchenko [12]. They have been studied by Deguchi in $[19,20]$. 


\section{The dimension of degenerate subspaces in the eight-vertex model.}

The transfer matrix $T(v)$ has the property that it satisfies the famous $T Q$ equation derived in [1]

$$
T(v) Q(v)=[\rho h(v-\eta)]^{N} Q(v+2 \eta)+[\rho h(v+\eta)]^{N} Q(v-2 \eta)
$$

where

$$
h(v)=\Theta(0) \Theta(v) H(v) .
$$

and $\Theta(v)$ and $H(v)$ are the standard Jacobi theta functions. It is important to recognize that the matrix $Q(v)$ in (3.1) is not unique. The original $Q$ matrix was discovered [1] in 1972 under the condition (1.1) and has been further studied in detail in [10]. In 1973 Baxter constructed [6] a second $Q$-matrix which is different from that of [1] and is defined for generic values of the crossing parameter $\eta$. To distinguish between these two rather different matrices we denote them as $Q_{72}$ and $Q_{73}$. Both matrices have the form

$$
Q(v)=Q_{R}(v) Q_{R}^{-1}\left(v_{0}\right)
$$

where $Q_{R, 72}$ is only defined when (1.1) holds. Like the transfer-matrix $T(v)$ the matrix $Q_{R}(v)$ is the trace of a product of local matrices which are in this case of size $L \times L$

$$
\left[Q_{R}(v)\right]_{\alpha \mid \beta}=\operatorname{Tr} S_{R}\left(\alpha_{1}, \beta_{1}\right) S_{R}\left(\alpha_{2}, \beta_{2}\right) \cdots S_{R}\left(\alpha_{N}, \beta_{N}\right)
$$

where $\alpha_{j}$ and $\beta_{j}= \pm 1$ and for the case $m_{2}=0$ in (1.1)

$$
\begin{aligned}
& S_{R}(\alpha, \beta)(v)_{k, l}=\delta_{k+1, l} \quad u^{\alpha}(v+K-2 k \eta) \tau_{-k, \beta}+\delta_{k, l+1} \quad u^{\alpha}(v+K+2 l \eta) \tau_{l, \beta}+ \\
& \delta_{k, 1} \delta_{l, 1} u^{\alpha}(v+K) \tau_{0, \beta} \quad+\delta_{k, L} \delta_{l, L} u^{\alpha}(v+K+2 L \eta) \tau_{L, \beta}
\end{aligned}
$$

for $1<k \leq L, 1<l \leq L$ and where

$$
u^{+}(v)=H(v) \quad u^{-}(v)=\Theta(v)
$$

We have shown in [10] that $Q_{R, 72}(v)$ has the (quasi)periodicity properties

$$
Q_{R, 72}(v+2 K)=S Q_{R, 72}(v)
$$

where

$$
S=\sigma_{3} \otimes \sigma_{3} \otimes \cdots \otimes \sigma_{3}
$$

and

$$
Q_{R, 72}\left(v+2 i K^{\prime}\right)=q^{-N} \exp \left(\frac{-i \pi N v}{K}\right) Q_{R, 72}(v)
$$

$Q_{72}$ can be formed in accordance with (3.3) only if $Q_{R, 72}^{-1}$ exists.

Whether $Q_{R, 72}^{-1}$ exists depends on the size $N$ of the spin chain. If $N$ is so large that exact complete strings could exist then as we found in [10]

$Q_{R, 72}^{-1}$ does NOT EXIST for real $\eta$ if $m_{1}$ is even and $L$ is odd.

It exists only for odd $m_{1}$ and even or odd $L$. 
It follows that for odd $m_{1}$ and even or odd $L$ the matrix $Q_{72}$ exits. It can be shown that it commutes with $T$. From (3.7) and (3.8) it follows that its root structure differs from that of $Q_{73}$. It is shown in [10] that

$$
\begin{gathered}
Q_{72}(v)=\hat{\mathscr{K}}\left(q ; v_{k}\right) \exp \left(i\left(n_{B}-v\right) \pi v / 2 K\right) \prod_{j=1}^{n_{B}} h\left(v-v_{j}^{B}\right) \\
\times \prod_{j=1}^{n_{L}} H\left(v-i w_{j}\right) H\left(v-i w_{j}-2 \eta\right) \cdots H\left(v-i w_{j}-2(L-1) \eta\right) \\
2 n_{B}+L n_{L}=N .
\end{gathered}
$$

$n_{B}$ is the number of Bethe roots $v_{k}$ and $n_{L}$ the number of exact $Q$-strings of length $L$.

Note that $n_{L}$ is always even.

$Q_{72}$ satisfies a functional relation conjectured in [10] and proven for $L=2$ in [22]: ${ }^{1}$

For $N$ even and either $L$ even or $L$ and $m_{1}$ odd

$$
\begin{aligned}
& e^{-N \pi i v / 2 K} Q_{72}\left(v-i K^{\prime}\right) \\
= & A \sum_{l=0}^{L-1} h^{N}(v-(2 l+1) \eta) \frac{Q_{72}(v)}{Q_{72}(v-2 l \eta) Q_{72}(v-2(l+1) \eta)}
\end{aligned}
$$

where $A$ is a normalizing constant matrix independent of $v$ that commutes with $Q_{72}$.

(3.11) determines the complete set of zeros of each eigenvalue of $Q_{72}$. So it delivers more information than Bethe' equations (which are contained in (3.11)). It determines the regular roots as well as the roots appearing in exact strings: If there exists an eigenvalue of $Q_{72}$ having $n_{L}$ exact strings in its set of zeros with string centers

$$
v_{c_{1}}, \cdots, v_{c_{n_{L}}}
$$

then there exist eigenvalues of $Q_{72}$ having the same regular roots and strings with centers

$$
v_{c_{1}}+\varepsilon_{1} i K^{\prime}, \cdots, v_{n_{L}}+\varepsilon_{n_{L}} i K^{\prime}
$$

for all $2^{n_{L}}$ sets

$$
\varepsilon_{i}=0,1, \quad i=1, \cdots, n_{L}
$$

It follows [18]: The eigenspace of a degenerate eigenvalue of $T$ has the dimension

$$
2^{n_{L}} \quad \text { or } \quad 2^{n_{L}-1}
$$

It is the purpose of the following sections to construct all eigenvectors of $T$ in these degenerate subspaces.

We conclude this section with three remarks:

1 .

Recently a $Q$-matrix has been found [21] for even $N$ which has the same properties as $Q_{72}$ and which exists for $\eta=2 m_{1} K / L$ where $Q_{72}$ does not exist.

\footnotetext{
${ }^{1}$ It is not sufficient to prove this functional equation in the subspace of $R$-invariant states [14]. It is important to show its validity in the degenerate subspaces of $T$.
} 
2.

The matrix $Q_{R}(u)$ defined by (3.4)-(3.6) is which is the definition given literally in [1] only satisfies the $T Q_{R}$ equation (C22) of [1] when (1.1) holds with $m_{2}=0$. To extend the working of [1] to the case $m_{2} \neq 0$ the definitions of all theta functions must be modified as done in [6] for the related root of unity condition (1.2). The required modification is

$$
\begin{aligned}
\tilde{\Theta}(v) & =\Theta(v) \exp \left[i \pi m_{2}(v-K)^{2} /(8 K L \eta)\right] \\
\tilde{H}(v) & =H(v) \exp \left[i \pi m_{2}(v-K)^{2} /(8 K L \eta)\right]
\end{aligned}
$$

With the replacements in (3.6) of $\Theta(v) \rightarrow \tilde{\Theta}(v)$ and $H(v) \rightarrow \tilde{H}(v)$ the matrix $Q_{72}(v)$ of (3.3) will satisfy the $T Q$ equation (3.1) whenever it exists for all $\eta$ which satisfy (1.1).

3.

In [14] it is claimed in equ. (A.33) that (in Baxter's notation)

$$
Q_{72}\left(v+i K^{\prime}\right)=q^{-N / 4} \exp (-N \pi v / 2 K) R Q_{72}(v)
$$

where

$$
R=\sigma_{1} \otimes \sigma_{1} \otimes \cdots \otimes \sigma_{1}
$$

However, the relation (3.16) cannot possibly hold because, if we recall that the eigenvectors of $Q_{72}(v)$ are independent of $v$, we see that (3.16) would imply that all eigenvectors of $Q_{72}(v)$ would be eigenvectors of $R$ which we demonstrated in [10] is not the case.

To isolate more explicitly the reason why (3.16) fails to hold we note that it follows from the quasiperiodicity properties of $H(v)$ and $\Theta(v)$ that for $m_{2}=0$ from (3.4)-(3.6)

$$
\begin{aligned}
& S_{R}( \pm, \beta)_{k, k+1}\left(v+i K^{\prime}\right)=f(v) \exp (i \pi k \eta / K) R S_{R}( \pm, \beta)_{k, k+1}(v) \\
& S_{R}( \pm, \beta)_{k+1, k}\left(v+i K^{\prime}\right)=f(v) \exp (-i \pi k \eta / K) R S_{R}( \pm, \beta)_{k+1, k}(v) \\
& S_{R}( \pm, \beta)_{1,1}\left(v+i K^{\prime}\right)=f(v) R S_{R}( \pm, \beta)_{1,1}(v) \\
& S_{R}( \pm, \beta)_{L, L}\left(v+i K^{\prime}\right)=(-1)^{m_{1}} f(v) R S_{R}( \pm, \beta)_{L, L}(v)
\end{aligned}
$$

where

$$
f(v)=q^{-1 / 4} \exp \left(-\frac{i \pi v}{2 K}\right)
$$

The relation (3.16) will follow only if $(-1)^{m_{1}}=1$ which requires that $m_{1}$ be even. But we found in [10] that if $m_{1}$ is even then when the number of lattice sites $N$ is sufficiently large that $Q_{R}(v)$ is singular for all $u$ and that $Q_{72}(v)$ as given by (3.3) does not exist. Consequently (3.16) fails to hold for the matrix $Q_{72}$.

\subsection{The string-free eigenstates of $T$.}

Here we state the important result derived in [11]: If the crossing parameter is restricted to (1.2) with $m_{2}=0$ and $N$ is even there are eigenstates of the transfer matrix $T$ given by

$$
\Psi_{m}=\sum_{l=0}^{L-1} e^{2 \pi i m l / L} B_{l+1, l-1}\left(\lambda_{1}\right) \cdots B_{l+n, l-n}\left(\lambda_{n}\right) \Omega_{N}^{l-n}
$$


where $\lambda_{1}, \cdots, \lambda_{n}$ are chosen to satisfy

$$
\frac{h^{N}\left(\lambda_{j}+\eta\right)}{h^{N}\left(\lambda_{j}-\eta\right)}=e^{-4 \pi i m / L} \prod_{k=1, k \neq j}^{n} \frac{h\left(\lambda_{j}-\lambda_{k}+2 \eta\right)}{h\left(\lambda_{j}-\lambda_{k}-2 \eta\right)} .
$$

with $N=2 n+$ integer $\times L$ and

$$
\begin{gathered}
\Omega_{N}^{l}=\omega_{1}^{l} \otimes \cdots \otimes \omega_{N}^{l} \\
\omega_{n}^{l}=\left(\begin{array}{c}
H(s+2(n+l) \eta-\eta) \\
\Theta(s+2(n+l) \eta-\eta)
\end{array}\right)
\end{gathered}
$$

We call the set $\lambda_{1}, \cdots, \lambda_{n}$ which solves the Bethe-equations (3.20) regular Bethe-roots. The $B_{k, l^{-}}$ operators are elements of the gauge transformed monodromy matrix

$$
\mathscr{T}_{k, l}=M_{k}^{-1}(\lambda) \mathscr{T}(\lambda) M_{l}(\lambda)=\left(\begin{array}{ll}
A_{k, l} & B_{k, l} \\
C_{k, l} & D_{k, l}
\end{array}\right) .
$$

As mentioned above the state vectors (3.19) give all singlet states as well as a subset of each degenerate multiplet of states.

\subsection{Eigenstates of $T$ with strings.}

The remaining much larger set of eigenvectors of $T$ has been obtained in [18]. These eigenvectors are given by

$$
\Psi=\sum_{l=0}^{L-1} \omega^{l} B_{l}^{L, 1}\left(\lambda_{c_{1}}\right) \ldots B_{l}^{L, 1}\left(\lambda_{c_{m}}\right) \prod_{m=L_{s}+1}^{n} B_{l+m, l-m}\left(\lambda_{m}\right) \Omega_{N}^{l-n}
$$

where $\omega=\exp (2 \pi i m l / L)$ and the contribution of an exact $L$-string is

$$
B_{l}^{L, 1}\left(\lambda_{c}\right)=\sum_{j=1}^{L_{s}} B_{l+1, l-1}\left(\lambda_{1}\right) \cdots\left(\frac{\partial B_{l+j, l-j}}{\partial \eta}\left(\lambda_{j}\right)-\hat{Z}_{j} \frac{\partial B_{l+j, l-j}}{\partial \lambda}\left(\lambda_{j}\right)\right) \cdots B_{l+L_{s}, l-L_{s}}\left(\lambda_{L_{s}}\right)
$$

and where $\lambda_{c_{i}}$ are the centers of strings

$$
\lambda_{k}=\lambda_{c}-2(k-1) \eta \quad k=1, \cdots, L_{s}
$$

We shall refer to (3.24) as B-string operator which creates the B-string (3.25). The string centers are free parameters. The string length $L_{s}$ is for odd $L$

$$
L_{s}=L
$$

and for even $L$

$$
L_{s}=L / 2
$$

The key of our method is the function $\hat{Z}_{j}$ in (3.24). Its definition is

$$
\begin{gathered}
\hat{Z}_{j}\left(\lambda_{c}\right)=\hat{Z}_{1}\left(\lambda_{c}-(j-1) 2 \eta\right) \\
\hat{Z}_{1}=-2 \frac{\sum_{k=0}^{L_{s}-1} k \frac{\omega^{-2(k+1)} \rho_{k+1}}{P_{k} P_{k+1}}}{\sum_{k=0}^{L_{s}-1} \frac{\omega^{-2(k+1)} \rho_{k+1}}{P_{k} P_{k+1}}}
\end{gathered}
$$




$$
\rho_{k}=h^{N}\left(\lambda_{c}-(2 k-1) \eta\right)
$$

and

$$
P_{k}=\prod_{m=L_{s}+1}^{n} h\left(\lambda_{c}-\lambda_{m}-2 k \eta\right)
$$

$\omega=e^{2 \pi i m / L}$ is a Lth root of unity. The constants $\lambda_{m}$ in (3.29) are the regular Bethe-roots of the state under consideration. We see that each string operator depends on its string center $\lambda_{c}$, on $s, t$ and on all regular roots defining the eigenvalue of $T$.

We briefly describe how the result (3.23)-(3.29) has been derived. The attempt to generate the missing eigenstates by adding a complete exact string

$$
\lambda_{k}=\lambda_{c}-2(k-1) \eta, \quad k=1, \cdots L_{s}
$$

to the set of Bethe roots fails because

$$
B_{l+1, l-1}\left(\lambda_{1}\right) \cdots B_{l+L_{s}, l-L_{s}}\left(\lambda_{L_{s}}\right)=0
$$

To circumvent this problem we insert instead of (3.30) the expression

$$
\lambda_{k}=\lambda_{c}-2(k-1) \eta-\hat{Z}_{k}\left(\lambda_{c}\right) \varepsilon, \quad k=1, \cdots L_{s}
$$

and perform the limit $\varepsilon \rightarrow 0$. The $O(\varepsilon)$ term gives the desired result.The function $\hat{Z}_{k}\left(\lambda_{c}\right)$ is an essential ingredient of our method.

We follow the same path as Takhtadzhan and Faddeev [11]: Let $\Psi$ be the candidate for an eigenstate of $T$ and

$$
T \Psi=\underbrace{t(\lambda) \Psi}_{\text {wanted term }}+\underbrace{\text { additional terms }}_{\text {unwanted }}
$$

In the work of [11] the unwanted terms can be removed by adjusting the set of free parameters $\lambda_{1}, \cdots, \lambda_{n}$ to satisfy " Bethe's equations". In our case a first set of unwanted terms is removed by again invoking Bethe's equations to determine $\lambda_{1}, \cdots \lambda_{n}$.

The remaining second set of unwanted terms is removed by appropriately choosing the functions $\hat{Z}_{k}\left(\lambda_{c}\right)$ which leads to

$$
\omega\left(\hat{Z}_{k+1}-\hat{Z}_{k}-2\right) \rho_{k-1} P_{k}=\omega^{-1}\left(\hat{Z}_{k}-\hat{Z}_{k-1}-2\right) \rho_{k} P_{k-2}
$$

where $\rho$ and $P_{k}$ and are defined in (3.28) and (3.29). The solution of (3.34) is (3.27).

The total number of operators $B$ building an eigenvector is restricted by

$$
2\left(n_{B}+n_{s}\right)+r L=N
$$

where $n_{B}$ is the number of regular roots and $n_{s}$ is the number of roots belonging to $B$-strings. It is important to note that the integer $r$ may be positive and negative. It follows that there is no restriction on the number of $B$-string-operators in a state vector. To understand the role of B-strings we note that the analytical expression for eigenstates of the eight-vertex model without strings given by equ. (3.19) depends on two free parameters $s, t$. For degenerate eigenstates which form a space of dimension $d$ a subspace of dimension $d_{0}<d$ can be constructed by the variation of $s$ and $t$ without 
applying B-string operators (3.24) . Detailed numerical studies have revealed that the variation of $s, t$ will give only for very small d (e.g. $d=2$ ) the full degenerate eigenspace. In all other cases one has to add B-string operators with the additional freedom to choose the string center to generate the full subspace. After this is achieved by adding a certain number of B-strings (the exact number depends on the system size $N$ and the value of $\eta$ ) the addition of more and more B-strings will only map this subspace into itself. In particular adding B-strings to a singlet state with $n_{B}=N / 2$ Bethe-roots does not destroy this state but reproduces it.

It is obvious that the B-string operators (3.24) play the role of symmetry operators in the eigenspaces of the transfer matrix. As they generate for each eigenvalue the full degenerate subspace they must contain all information about the hidden symmetry algebra. The properties described above raise the possibility that the B-string operators (3.24) are related to elliptic current operators in cyclic representations. We expect that the $q \rightarrow 0$ limit of this algebra is the $s l_{2}$-loop algebra which has been found in [15] and [16] to describe the symmetry of the six-vertex model. This suggests that the symmetry is related to $U_{q}\left(\widehat{s l_{2}}\right)$. To our knowledge the theory of cyclic representations of $U_{q}\left(\widehat{s l_{2}}\right)$ is still undeveloped. ${ }^{2}$

\section{Acknowledgements}

We wish to thank the organizers of this Solvay conference for the opportunity to participate in the celebration of the 75th anniversary of Bethe's ansatz.

\section{References}

[1] R.J. Baxter, Partition Function of the Eight-Vertex Lattice Model, Ann. Phys. 70, (1972) 193-228.

[2] H.A. Bethe, Zur Theorie der Metalle. Z. Physik 71,205(1931).

[3] E.H.Lieb, Exact Solution of the problem of the entropy of two-dimensional ice, Phys. Rev. Letts. 18 (1967) 692; Exact Solution of the F model of an antiferroelectric,Phys. Rev. Letts. 18 (1967) 1046; Exact solution of the two-dimensional Slater KDP model of a ferroelectric, Phys. Rev. Letts. 19 (1967) 108-110; Residual Entropy of Square Ice, Phys. Rev.162, 162-172 (1967).

[4] B. Sutherland, Exact solution of a two-dimensional model for hydrogen bonded crystals, Phys. Rev. Letts. 19 (1967) 103-104;

B. Sutherland, C.N. Yang and C.P. Yang, Exact solution of a model of two-dimensional ferroelectrics in an arbitrary external electric field, Phys. Rev. Letts. 19 (1967) 588-591.

[5] C.N. Yang and C.P. Yang, One-Dimensional Chain of Anisotropic Spin-Spin Interactions.I. Proof of Bethe's Hypothesis for Ground State in a Finite System, Phys. Rev. 150 (1066) 321-327; One-Dimensional Chain of Anisotropic Spin-Spin Interactions. II. Properties of the Ground-State Energy Per Lattice Site for an Infinite System , Phys. Rev. 150 (1966) 327-339.

[6] R.J. Baxter, Eight-Vertex Model in Lattice Statistics and One-Dimensional Anisotropic Heisenberg Chain I. Some Fundamental Eigenvectors, Ann. Phys. 76 (1973) 1-23.

\footnotetext{
${ }^{2}$ We thank V. Chari, A. Kirillov and V. Kac for this information.
} 
[7] R.J. Baxter, Eight-Vertex Model in Lattice Statistics and One-Dimensional Anisotropic Heisenberg Chain II. Equivalence to a Generalized Ice-type Lattice Model, Ann. Phys. 76 (1973) 25-47.

[8] R.J. Baxter, Eight-Vertex Model in Lattice Statistics and One-Dimensional Anisotropic Heisenberg Chain III. Eigenvectors of the Transfer Matrix and Hamiltonian, Ann. Phys. 76 (1973) 48-70.

[9] R. J. Baxter, Exactly Solved Models in Statistical Mechanics, Academic Press. London (1982).

[10] K. Fabricius and B.M. McCoy, New Developments in the Eight Vertex Model, J. Stat. Phys. 111 (2003) 323-337.

[11] L.A. Takhtadzhan and L.D. Faddeev, The Quantum Method of the Inverse Problem and the Heisenberg XYZ Model, Russian Math. Surveys 34:5 (1979) 11 - 68; translated from Uspekhi Mat. Nauk 34:5 (1979) $13-63$.

[12] G. Felder and A. Varchenko, Algebraic Bethe ansatz for the elliptic quantum group $E_{\tau . \eta}\left(s l_{2}\right)$, Nucl. Phys. B480 (1996) 485-503.

[13] R.J. Baxter, Completeness of the Bethe Ansatz for the Six and Eight-Vertex Models . J.Stat. Phys. 108,1-48 (2002)

[14] V.V. Bazhanov and V.V. Mangazeev, Analytic theory of the eight-vertex model . arXiv:hep-th/0609153

[15] T. Deguchi, K. Fabricius and B.M. McCoy, The sl 2 Loop Algebra Symmetry of the Six-Vertex Model at Roots of Unity, J. Stat. Phys. 102 (2001) 701.

[16] K. Fabricius and B. McCoy, Evaluation Parameters and Bethe Roots for the Six-Vertex Model at Roots of Unity . MathPhys Odyssey 2001, Progress in Mathematical Physics 23. ed. M. Kashiwara and T. Miwa (Birkhäuser Boston 2002) 119-144.

[17] T. Deguchi, XXZ Bethe states as highest weight vectors of the sl 2 loop algebra at roots of unity, arXiv:cond-mat/0503564.

[18] K. Fabricius and Barry McCoy, An elliptic current operator for the eight-vertex model . J.Phys.A: Math.Gen.39 (2006) 14869-14886.

[19] T. Deguchi, The 8 V CSOS model and the sl 2 loop algebra symmetry of the six vertex model at roots of unity, Int. J. Mod. Phys. B16 (2002) 1899-1905.

[20] T. Deguchi, Construction of some missing eigenvectors of the XYZ spin chain at the discrete coupling constants and the exponentially large spectral degeneracy of the transfer matrix, J. Phys. A35 (2002) 879-895.

[21] K. Fabricius, A new Q-Matrix in the Eight Vertex Model, arXiv:cond-mat/0610481.

[22] K. Fabricius and Barry McCoy, Functional Equations and Fusion Matrices for the Eight Vertex Model, RIMS 40, 905 (2004) 